\title{
Sharp global nonlinear stability for a fluid overlying a highly porous material
}

\author{
By ANTONY A. HILL ${ }^{1}$ AND MAGDA CARR ${ }^{2}$ \\ ${ }^{1}$ School of Mathematical Sciences, University of Nottingham, Nottingham, \\ $N G 7$ 2RD, UK \\ ${ }^{2}$ School of Mathematics and Statistics, University of St Andrews, St Andrews, \\ KY16 9SS, UK
}

The stability of convection in a two-layer system in which a layer of fluid with a temperature dependent viscosity overlies and saturates a highly porous material is studied. Due to the difficulties associated with incorporating the nonlinear advection term in the Navier Stokes equations into a stability analysis, previous literature on fluid/porous thermal convection has modelled the fluid using the linear Stokes equations. This paper derives global stability for the full nonlinear system, by utilising a model proposed by Ladyzhenskaya. The nonlinear stability boundaries are shown to be sharp when compared with the linear instability thresholds. Keywords: superposed porous-fluid convection; temperature dependent
viscosity; energy method

\section{Introduction}

Thermal convection within a two-layer system constructed by a layer of fluid overlying a porous material saturated with the same fluid has numerous geophysical and industrial applications, such as the manufacturing of composite materials used in the aircraft and automobile industries, flow of water under the Earth's surface, flow of oil in underground reservoirs and growing of compound films in thermal chemical vapor deposition reactors. A detailed review is given by Nield \& Bejan (2006), with current highly relevant literature including Chen \& Chen (1988), Ewing (1998), Blest et al. (1999), Straughan (2002, 2008), Carr (2004), Chang (2004, 2005, 2006), Hirata et al. (2007), Hoppe et al. (2007), Mu \& Xu (2007) and Hill \& Straughan (2009).

Assessing the onset and type of convection is crucial in understanding and controlling these geophysical and industrial processes. This is achieved by analyzing both the linear instability and nonlinear stability thresholds of the governing model. Comparing these thresholds allows the assessment of the suitability of linear theory to predict the physics of the onset of convection. The derivation of sharp unconditional stability thresholds is particularly physically useful due to the lack of restrictions on the initial data (Straughan 2004).

Nonlinear energy stability analyses of thermal fluid/porous systems are not widespread in the current literature, with the only previous work being that of Payne \& Straughan (1998) and Hill \& Straughan (2009). In both these papers, due to the difficulties associated with incorporating the nonlinear $\mathbf{v} \cdot \nabla \mathbf{v}$ advection term 
in the Navier-Stokes equations into a stability analysis, the fluid is modelled using the linear Stokes equations.

This paper utilitses a model proposed by Ladyzhenskaya (Ladyzhenskaya 1967, 1968, 1969; Straughan 2002, 2004, 2008), which is used as an alternative to NavierStokes. This allows for the development of an unconditional nonlinear energy stability analysis for thermal convection with temperature dependent viscosity in a fluid/porous system, without the need to remove the nonlinear advection term $\mathbf{v} \cdot \nabla \mathbf{v}$. It is important to note that the viscosity of a liquid is usually strongly dependent on temperature (cf. Capone \& Gentile 1994, 1995; Galiano 2000). Convection problems for which the viscosity or conductivity is a function of temperature has received much recent attention in the literature (see e.g. Payne \& Straughan 2000; Shevtsova et al. 2001; Manga et al. 2001), making this work particularly timely.

The stability calculations required to construct the neutral curves involve determining eigenvalues and eigenfunctions, where the associated eigenvalue problems are not solvable analytically. The results are derived numerically using the Chebyshev tau - QZ method (Dongarra et al. 1996), which is a spectral method coupled with the QZ algorithm. All numerical results were checked by varying the number of polynomials to verify convergence. Standard indicial notation is employed throughout and $\mathbf{k}=(0,0,1)$.

\section{Formation of the problem}

Consider a fluid occupying the three-dimensional layer $\left\{(x, y) \in \mathbb{R}^{2}\right\} \times\{z \in(0, d)\}$ and saturating an underlying homogeneous porous medium $\left\{(x, y) \in \mathbb{R}^{2}\right\} \times\{z \in$ $\left.\left(-d_{m}, 0\right)\right\}$. The interface between the saturated porous medium and the fluid is at $z=0$.

We assume that the dynamic viscosity $\mu$ has a linear temperature dependence of the form

$$
\mu(T)=\mu_{0}\left(1-\gamma\left(T-T_{L}\right)\right),
$$

for a constant $\gamma>0$, where $T, \mu_{0}$ and $T_{L}$ are temperature and reference viscosity and temperature values, respectively. Although we only consider liquids which have a viscosity which decreases with increasing temperature, the analysis can be easily generalized to a more general viscosity-temperature relationship. The governing model for the fluid layer we select is

$$
\begin{aligned}
\rho_{0}\left(\frac{\partial v_{i}}{\partial t}+v_{j} \frac{\partial v_{i}}{\partial x_{j}}\right) & =-\frac{\partial p}{\partial x_{i}}+2 \frac{\partial}{\partial x_{j}}\left[\left(\mu(T)+\mu_{1}|\mathbf{D}|\right) D_{i j}\right] \\
& -g \rho_{0} k_{i}\left(1-\alpha\left(T-T_{L}\right)\right), \\
\frac{\partial v_{i}}{\partial x_{i}} & =0, \\
\frac{\partial T}{\partial t}+v_{j} \frac{\partial T}{\partial x_{j}} & =\frac{\kappa_{f}}{\left(\rho_{0} c_{p}\right)_{f}} \nabla^{2} T
\end{aligned}
$$

(Straughan 2002, 2004; Antontsev et al. 2001) where $v_{i}, p, t$ and $\rho_{0}$ are velocity, pressure, time and reference density; and $\kappa_{f}, g, c_{p}$ and $\alpha$ are the thermal conductivity, acceleration due to gravity, specific heat at a constant pressure and coefficient 
of thermal expansion. A variation of this model was suggested by Ladyzhensakaya $(1967,1968,1969)$ as an alternative to the Navier-Stokes equations, and is a generalisation of a well known model in viscoelasticity (Antontsev et al. 2001). The parameter $\mu_{1}>0$ is a constant, $D_{i j}=\left(v_{i, j}+v_{j, i}\right) / 2$ and $|\mathbf{D}|=\sqrt{D_{i j} D_{i j}}$. The subscripts (or superscripts) $f$ and $m$ denote the fluid and porous layers respectively.

In the porous medium we assume a high porosity $\phi>0.75$, such that the governing equations are given by

$$
\begin{aligned}
\frac{\rho_{0}}{\phi}\left(\frac{\partial v_{i}^{m}}{\partial t}+\frac{1}{\phi} v_{j}^{m} \frac{\partial v_{i}^{m}}{\partial x_{j}}\right)= & -\frac{\partial p^{m}}{\partial x_{i}}+\frac{2}{\phi} \frac{\partial}{\partial x_{j}}\left[\left(\mu\left(T^{m}\right)+\mu_{1}\left|\mathbf{D}^{m}\right|\right) D_{i j}^{m}\right] \\
& -\frac{\mu\left(T^{m}\right)}{K} v_{i}^{m}-g \rho_{0} k_{i}\left(1-\alpha\left(T^{m}-T_{L}\right)\right), \\
\frac{\partial v_{i}^{m}}{\partial x_{i}}= & 0, \\
\frac{\left(\rho_{0} c_{p}\right)^{*}}{\left(\rho_{0} c_{p}\right)_{f}} \frac{\partial T^{m}}{\partial t}+v_{j}^{m} \frac{\partial T^{m}}{\partial x_{j}}= & \frac{\kappa^{*}}{\left(\rho_{0} c_{p}\right)_{f}} \nabla^{2} T^{m},
\end{aligned}
$$

where the variables $v_{i}^{m}, p^{m}, T^{m}$ and $K$ are the velocity, pressure, temperature and permeability, respectively. The starred quantities are defined in terms of the fluid and porous variables such that $S^{*}=\phi S_{f}+(1-\phi) S_{m}$, where $S^{*}=\kappa^{*}$ or $\left(\rho_{0} c_{p}\right)^{*}$. A comprehensive discussion of the variances and various physical attributes of modelling transport through porous media is given in Alazmi \& Vafai (2000).

The temperatures at the upper and lower boundaries are held fixed at $T_{U}$ and $T_{L}$, respectively, with continuity of temperature, velocity and heat flux at the interface $z=0$. The remaining boundary conditions at $z=0$ are the continuity of normal stresses

$$
-p+2\left(\mu(T)+\mu_{1}|\mathbf{D}|\right) D_{33}=-p^{m}+\frac{2}{\phi}\left(\mu\left(T^{m}\right)+\mu_{1}\left|\mathbf{D}^{m}\right|\right) D_{33}^{m},
$$

and tangential stresses

$$
\left(\mu(T)+\mu_{1}|\mathbf{D}|\right) D_{\beta 3}=\frac{1}{\phi}\left(\mu\left(T^{m}\right)+\mu_{1}\left|\mathbf{D}^{m}\right|\right) D_{\beta 3}^{m},
$$

for $\beta=1,2$. The derivation of appropriate boundary conditions at the fluid/porous interface is non-trivial, cf. Vafai \& Thiyagaraja (1987), Alazmi \& Vafai (2001), Vafai (2005).

Under these boundary conditions, the governing equations $(2.1)-(2.2)$ admit a steady state solution in which the velocity field is zero and

$$
\begin{aligned}
\bar{T} & =T_{L}-\frac{\epsilon_{T}\left(T_{L}-T_{U}\right)}{\hat{d}+\epsilon_{T}}-\frac{\left(T_{L}-T_{U}\right)}{d_{m}\left(\hat{d}+\epsilon_{T}\right)} z, \quad z \in(0, d), \\
\bar{T}^{m} & =T_{L}-\frac{\epsilon_{T}\left(T_{L}-T_{U}\right)}{\hat{d}+\epsilon_{T}}-\frac{\epsilon_{T}\left(T_{L}-T_{U}\right)}{d_{m}\left(\hat{d}+\epsilon_{T}\right)} z, \quad z \in\left(-d_{m}, 0\right),
\end{aligned}
$$

where $\epsilon_{T}=\tau_{f} / \tau_{m}, \tau_{f}=\kappa_{f} /\left(\rho_{0} c_{p}\right)_{f}, \tau_{m}=\kappa^{*} /\left(\rho_{0} c_{p}\right)_{f}$ and $\hat{d}=d / d_{m}$, with the overbar denoting the steady state. To study the stability of the steady state we 
introduce the perturbations $\left(u_{i}, \theta, \pi, u_{i}^{m}, \theta^{m}, \pi^{m}\right)$, where $d_{i j}=\left(u_{i, j}+u_{j, i}\right) / 2$, and non-dimensionalisize with the scalings

$$
\begin{aligned}
& u_{i}=\frac{\mu_{0}}{\rho_{0} d} u_{i}^{*}, \quad \pi=\frac{\mu_{0}^{2}}{\rho_{0} d^{2}} \pi^{*}, \quad \theta=\theta^{*} \sqrt{\frac{\mu_{0}^{3}\left(T_{L}-T_{U}\right)}{\rho_{0}^{3} g \alpha d^{3} \tau_{f}}}, \quad x_{i}=d x_{i}^{*}, \\
& t=\frac{\rho_{0} d^{2}}{\mu_{0}} t^{*}, \quad R=\sqrt{\frac{g \alpha \rho_{0} d^{3}\left(T_{L}-T_{U}\right)}{\mu_{0} \tau_{f}}}
\end{aligned}
$$

where $R_{a}=R^{2}$ is the fluid Rayleigh number. By replacing $d$ and $\tau_{f}$ by $d_{m}$ and $\tau_{m}$, respectively, the porous layer scalings follow analogously, where $R_{a}^{m}=\left(R^{m}\right)^{2}$ is the porous Rayleigh number. This yields the non-dimensional perturbation equations

$$
\begin{aligned}
\frac{\partial u_{i}}{\partial t}+u_{j} \frac{\partial u_{i}}{\partial x_{j}}= & -\frac{\partial \pi}{\partial x_{i}}+k_{i} R \theta-\frac{2 \Gamma P r}{R} \frac{\partial}{\partial x_{j}}\left(\theta d_{i j}\right)+2 \omega \frac{\partial}{\partial x_{j}}\left(|\mathbf{d}| d_{i j}\right) \\
& +2 \frac{\partial}{\partial x_{j}}\left(f_{1} d_{i j}\right) \\
\frac{\partial u_{i}}{\partial x_{i}}= & 0 \\
\operatorname{Pr}\left(\frac{\partial \theta}{\partial t}+u_{j} \frac{\partial \theta}{\partial x_{j}}\right)= & R M_{1} u_{3}+\nabla^{2} \theta
\end{aligned}
$$

in $\mathbb{R}^{2} \times(0,1) \times(0, \infty)$ with $f_{1}=1+\Gamma\left(M_{2}+M_{1} z\right), d_{i j}=\left(u_{i, j}+u_{j, i}\right) / 2$ and

$$
\begin{aligned}
\frac{1}{\phi} \frac{\partial u_{i}^{m}}{\partial t}+\frac{1}{\phi^{2}} u_{j}^{m} \frac{\partial u_{i}^{m}}{\partial x_{j}}= & -\frac{\partial \pi^{m}}{\partial x_{i}}+k_{i} R^{m} \theta^{m}-\frac{f_{2}}{\delta} u_{i}^{m}+\frac{2 \hat{d}^{2} \omega}{\phi} \frac{\partial}{\partial x_{j}}\left(\left|\mathbf{d}^{m}\right| d_{i j}^{m}\right) \\
& +\frac{2}{\phi} \frac{\partial}{\partial x_{j}}\left(f_{2} d_{i j}^{m}\right)+\frac{\Gamma P r \epsilon_{T}}{R^{m}}\left(\frac{1}{\delta} u_{i}^{m} \theta^{m}-\frac{2}{\phi} \frac{\partial}{\partial x_{j}}\left(\theta^{m} d_{i j}^{m}\right)\right) \\
\frac{\partial u_{i}^{m}}{\partial x_{i}}= & 0, \\
\operatorname{Pr}_{T}\left(G_{m} \frac{\partial \theta^{m}}{\partial t}+u_{j}^{m} \frac{\partial \theta^{m}}{\partial x_{j}}\right)= & R^{m} M_{2} u_{3}^{m}+\nabla^{2} \theta^{m},
\end{aligned}
$$

in $\mathbb{R}^{2} \times(-1,0) \times(0, \infty)$, with $f_{2}=1+\Gamma M_{2}(1+z), d_{i j}^{m}=\left(u_{i, j}^{m}+u_{j, i}^{m}\right) / 2$. The remaning parameters are the Prandtl number $\operatorname{Pr}=\mu_{0} /\left(\kappa_{f} \rho_{0}\right)$, Darcy number $\delta=$ $K / d_{m}^{2}, \omega=\mu_{1} /\left(\rho_{0} d^{2}\right), \Gamma=\gamma\left(T_{L}-T_{U}\right), G_{m}=\left(\rho_{0} c_{p}\right)^{*} /\left(\rho_{0} c_{p}\right)_{f}, M_{1}=\hat{d} /\left(\hat{d}+\epsilon_{T}\right)$ and $M_{2}=\epsilon_{T} /\left(\hat{d}+\epsilon_{T}\right)$.

\section{Linear Instability Analysis}

To proceed with a linear analysis, the nonlinear terms from (2.5) and (2.6) are discarded. We assume normal modes of the form

$$
u_{i}=u_{i}(z) \mathrm{e}^{\sigma t+i\left(a_{1} x+a_{2} y\right)}, \quad \pi=\pi(z) \mathrm{e}^{\sigma t+i\left(a_{1} x+a_{2} y\right)}, \quad \theta=\theta(z) \mathrm{e}^{\sigma t+i\left(a_{1} x+a_{2} y\right)},
$$

with analogous definitions in the porous medium. Taking the double curls of $(2.5)_{1}$ and $(2.6)_{1}$ to remove the pressure terms, where the third component is chosen, leads 
to the linearised equations

$$
\begin{aligned}
f_{1}\left(D^{2}-a^{2}\right)^{2} w+2 \Gamma M_{1}\left(D^{2}-a^{2}\right) D w-a^{2} R \theta & =\sigma\left(D^{2}-a^{2}\right) w \\
\left(D^{2}-a^{2}\right) \theta+R M_{1} w & =\operatorname{Pr} \sigma \theta \\
\frac{f_{2}}{\phi}\left(D^{2}-a_{m}^{2}-\frac{\phi}{\delta}\right)\left(D^{2}-a_{m}^{2}\right) w^{m} & +\frac{2 \Gamma M_{2}}{\phi}\left(D^{2}-a_{m}^{2}-\frac{\phi}{2 \delta}\right) D w^{m} \\
-a_{m}^{2} R^{m} \theta^{m} & =\frac{\sigma^{m}}{\phi}\left(D^{2}-a_{m}^{2}\right) w^{m} \\
\left(D^{2}-a_{m}^{2}\right) \theta^{m}+R^{m} M_{2} w^{m} & =\operatorname{Pr}^{m} \epsilon_{T} G_{m} \theta^{m}
\end{aligned}
$$

where $D=\mathrm{d} / \mathrm{d} z, a^{2}=a_{1}^{2}+a_{2}^{2}$ and $a_{m}^{2}=\left(a_{1}^{m}\right)^{2}+\left(a_{2}^{m}\right)^{2}$. The boundary conditions for the twelfth order system at $z=1$ are

$$
w=D w=\theta=0,
$$

and

$$
w^{m}=D w^{m}=\theta^{m}=0
$$

at $z=-1$. On the interface $z=0$, we have

$$
\begin{aligned}
& w=\hat{d} w, \quad D w=\hat{d}^{2} D w^{m}, \\
& \phi\left(D^{2}+a^{2}\right) w=\hat{d}^{3}\left(D^{2}+a_{m}^{2}\right) w^{m}, \quad \theta=\sqrt{\epsilon_{T} \hat{d}^{3}} \theta^{m}, \\
& D \theta=\sqrt{\frac{\hat{d}^{5}}{\epsilon_{T}}} D \theta^{m}
\end{aligned}
$$

and

$$
\begin{aligned}
f_{1}\left(D^{2}-3 a^{2}\right) D w+ & \Gamma M_{1}\left(D^{2}+a^{2}\right) w-\sigma D w=\frac{\hat{d}^{4} f_{2}}{\phi}\left(D^{2}-3 a_{m}^{2}\right) w^{m}+ \\
& \hat{d}^{4} \frac{\Gamma M_{2}}{\phi}\left(D^{2}+a_{m}^{2}\right) w^{m}-\frac{f_{2} \hat{d}^{4}}{\delta} D w^{m}-\frac{\hat{d}^{4} \sigma_{m}}{\phi} D w^{m} .
\end{aligned}
$$

The numerical results are presented in $\S 5$.

\section{Nonlinear Stability Analysis}

Let us define $\Omega_{f}$ and $\Omega_{m}$ to represent the period cells in the fluid and porous layers respectively, and introduce the notation of norm and inner product on the spaces $L^{2}\left(\Omega_{f}\right)$ and $L^{2}\left(\Omega_{m}\right)$, where

$$
\|f\|_{\alpha}^{2}=\int_{\Omega_{\alpha}} f_{i} f_{i} d \Omega_{\alpha}, \quad(f, g)_{\alpha}=\int_{\Omega_{\alpha}} f_{i} g_{i} d \Omega_{\alpha}, \quad \alpha=f, m .
$$

To obtain global nonlinear stability bounds in the stability measure $L^{2}\left(\Omega_{f}\right)$ we multiply equations $(2.5)_{1}$ and $(2.5)_{3}$ by $u_{i}$ and $\theta$ respectively, and integrate over 
the period cell. An analogous process is applied to $(2.6)_{1}$ and $(2.6)_{3}$. We may now define the functional $E(t)$ by

$$
2 E(t)=\|\mathbf{u}\|_{f}^{2}+\lambda_{1} \operatorname{Pr}\|\theta\|_{f}^{2}+\frac{\lambda_{2}}{\phi}\left\|\mathbf{u}^{m}\right\|_{m}^{2}+\lambda_{3} \epsilon_{T} G_{m} \operatorname{Pr}\left\|\theta^{m}\right\|_{m}^{2}
$$

for coupling parameters $\lambda_{1}, \lambda_{2}, \lambda_{3}>0$, such that

$$
\begin{aligned}
\frac{\mathrm{d} E}{\mathrm{~d} t}= & \left(u_{i},\left[-u_{j} u_{i, j}-\pi_{, i}+k_{i} R \theta-\frac{2 \Gamma \operatorname{Pr}}{R}\left(\theta d_{i j}\right)_{, j}+2 \omega\left(|\mathbf{d}| d_{i j}\right)_{, j}+2\left(f_{1} d_{i j}\right)_{, j}\right]\right)_{f} \\
& +\lambda_{1}\left(\theta,\left[-\operatorname{Pru}_{i} \theta_{, i}+R M_{1} w+\nabla^{2} \theta\right]\right)_{f}+\lambda_{2}\left(u_{i}^{m},\left[-\frac{1}{\phi^{2}} u_{j}^{m} u_{i, j}^{m}-\pi_{, i}^{m}\right.\right. \\
& +k_{i} R^{m} \theta^{m}-\frac{f_{2}}{\delta} u_{i}^{m}-\frac{2 \Gamma \operatorname{Pr} \epsilon_{T}}{\phi R^{m}}\left(\theta^{m} d_{i j}^{m}\right)_{, j}+\frac{\Gamma \operatorname{Pr} \epsilon_{T}}{\delta R^{m}} \theta^{m} u_{i}^{m}+\frac{2 \hat{d}^{2} \omega}{\phi}\left(\left|\mathbf{d}^{m}\right| d_{i j}^{m}\right)_{, j} \\
& \left.\left.+\frac{2}{\phi}\left(f_{2} d_{i j}^{m}\right)_{, j}\right]\right)_{m}+\lambda_{3}\left(\theta^{m},\left[-\epsilon_{T} \operatorname{Pru}_{i}^{m} \theta_{, i}^{m}+R^{m} M_{2} w^{m}+\nabla^{2} \theta^{m}\right]\right)_{m} .
\end{aligned}
$$

Utilising a similar approach to Hill \& Straughan (2009), the first and third terms on the right hand side of (4.1) are integrated by parts, and the nondimensionalised versions of boundary condtions (2.3) and (2.4) are employed to yield

$$
\begin{aligned}
& \left(u_{i},\left[-u_{j} u_{i, j}-\pi_{, i}-\frac{2 \Gamma P r}{R}\left(\theta d_{i j}\right)_{, j}+2 \omega\left(|\mathbf{d}| d_{i j}\right)_{, j}+2\left(f_{1} d_{i j}\right)_{, j}\right]\right)_{f} \\
& +\lambda_{2}\left(u_{i}^{m},\left[-\frac{1}{\phi^{2}} u_{j}^{m} u_{i, j}^{m}-\pi_{, i}^{m}-\frac{2 \Gamma P r \epsilon_{T}}{\phi R^{m}}\left(\theta^{m} d_{i j}^{m}\right)_{, j}+\frac{2 \hat{d}^{2} \omega}{\phi}\left(\left|\mathbf{d}^{m}\right| d_{i j}^{m}\right)_{, j}+\frac{2}{\phi}\left(d_{i j}^{m}\right)_{, j}\right]\right)_{m} \\
& \quad=\frac{1}{2} \int_{\Lambda}\left(|\mathbf{u}|^{2} w-\frac{\hat{d}^{3}}{\phi^{2}}\left|\mathbf{u}^{m}\right|^{2} w^{m}\right) d S-2 \omega \int_{\Omega_{f}}|\mathbf{d}|^{3} d \Omega_{f}-2 \int_{\Omega_{f}} f_{1}|\mathbf{d}|^{2} d \Omega_{f} \\
& \quad+\frac{2 \Gamma P r}{R} \int_{\Omega_{f}} \theta|\mathbf{d}|^{2} d \Omega_{f}-\frac{2 \hat{d}^{5} \omega}{\phi} \int_{\Omega_{m}}\left|\mathbf{d}^{m}\right|^{3} d \Omega_{m}-\frac{2 \hat{d}^{3}}{\phi} \int_{\Omega_{m}} f_{2}|\mathbf{d}|^{2} d \Omega_{m} \\
& \quad+\frac{2 \Gamma \operatorname{Pr} \epsilon_{T} \hat{d}^{3}}{\phi R^{m}} \int_{\Omega_{m}} \theta^{m}\left|\mathbf{d}^{m}\right|^{2} d \Omega_{m},
\end{aligned}
$$

where $\lambda_{2}=\hat{d}^{3}$, and $\Lambda$ represents the fluid/porous interface at $z=0$. Similarly, by integrating by parts and utilising the non-dimensionalised boundary conditions

$$
\begin{aligned}
\lambda_{1}\left(\theta,\left[-\operatorname{Pru}_{i} \theta_{, i}+\nabla^{2} \theta\right]\right)_{f} & +\lambda_{3}\left(\theta^{m},\left[-\operatorname{Pr}_{T} u_{i}^{m} \theta_{, i}^{m}+\nabla^{2} \theta^{m}\right]\right)_{m} \\
& =-\lambda\left(\|\nabla \theta\|_{f}^{2}+\hat{d}^{4}\left\|\nabla \theta^{m}\right\|_{m}^{2}\right)
\end{aligned}
$$

where $\lambda_{1}=\lambda$ and $\lambda_{3}=\lambda \hat{d}^{4}$. 
Combining these definitions it follows that

$$
\begin{aligned}
\frac{\mathrm{d} E}{\mathrm{~d} t}= & \frac{1}{2} \int_{\Lambda}\left(|\mathbf{u}|^{2} w-\frac{\hat{d}^{3}}{\phi^{2}}\left|\mathbf{u}^{m}\right|^{2} w^{m}\right) d S+R<\theta, w>-2 \omega \int_{\Omega_{f}}|\mathbf{d}|^{3} d \Omega_{f} \\
& -2 \int_{\Omega_{f}} f_{1}|\mathbf{d}|^{2} d \Omega_{f}+\frac{2 \Gamma P r}{R} \int_{\Omega_{f}} \theta|\mathbf{d}|^{2} d \Omega_{f}-\lambda\|\nabla \theta\|^{2}+\lambda R M_{1}<w, \theta> \\
& +\hat{d}^{3} R^{m}<\theta^{m}, u_{i}^{m}>-\frac{\hat{d}^{3}}{\delta} \int_{\Omega_{m}} f_{2}\left|\mathbf{u}^{m}\right|^{2} d \Omega_{m}+\frac{\Gamma P r \epsilon_{T} \hat{d}^{3}}{\delta R^{m}} \int_{\Omega_{m}} \theta^{m}\left|\mathbf{u}^{m}\right|^{2} d \Omega_{m} \\
& -\frac{2 \hat{d}^{5} \omega}{\phi} \int_{\Omega_{m}}\left|\mathbf{d}^{m}\right|^{3} d \Omega_{m}-\frac{2 \hat{d}^{3}}{\phi} \int_{\Omega_{m}} f_{2}\left|\mathbf{d}^{m}\right|^{2} d \Omega_{m} \\
& +\frac{2 \Gamma P r \epsilon_{T} \hat{d}^{3}}{\phi R^{m}} \int_{\Omega_{m}} \theta^{m}\left|\mathbf{d}^{m}\right|^{2} d \Omega_{m}+R^{m} \hat{d}^{4} M_{2} \lambda<w^{m}, \theta^{m}>-\lambda \hat{d}^{4}\left\|\nabla \theta^{m}\right\|^{2} .
\end{aligned}
$$

To address the cubic nonlinearities in (4.2) we introduce the $L^{3}$ norm $\|\cdot\|_{3}$. Multiplying $(2.5)_{3}$ and $(2.6)_{3}$ by $\theta^{2}$ and $\left(\theta^{m}\right)^{2}$, respectively, integrating over the period cell, and using Poincaré's inequality we find

$$
\begin{aligned}
\frac{\lambda_{4} P r}{3} \frac{d}{d t}\|\theta\|_{3}^{3}+ & \frac{\lambda_{5} \operatorname{Pr} \epsilon_{T} G_{m}}{3} \frac{d}{d t}\left\|\theta^{m}\right\|_{3}^{3} \leq \lambda_{4} R M_{1} \int_{\Omega_{f}} w \theta^{2}(\operatorname{sgn} \theta) d \Omega_{f} \\
& +\lambda_{4} \epsilon_{T}^{\frac{1}{2}} \hat{d}^{\frac{11}{2}} R M_{2} \int_{\Omega_{m}} w^{m}\left(\theta^{m}\right)^{2}\left(\operatorname{sgn} \theta^{m}\right) d \Omega_{m} \\
& -\frac{8 \pi^{2} \lambda_{4}}{9} \int_{\Omega_{f}}\left|\theta^{3}\right| d \Omega_{f}-\frac{8 \pi^{2} \lambda_{4} \epsilon_{T}^{\frac{1}{2}} \hat{d}^{\frac{11}{2}}}{9} \int_{\Omega_{m}}\left|\theta^{m}\right|^{3} d \Omega_{m}
\end{aligned}
$$

where $\lambda_{5}=\lambda_{4} \epsilon_{T}^{\frac{1}{2}} \hat{d}^{\frac{11}{2}}$ to ensure the removal of the boundary integrals (Hill \& Straughan 2009). We now use Young's inequality on the cubic integral terms in both (4.2) and (4.3), such that

$$
\int_{\Omega} Q_{1} Q_{2}^{2} d \Omega \leq \frac{c^{2}}{3}\left\|Q_{1}\right\|_{3}^{3}+\frac{1}{3 c}\left\|Q_{2}\right\|_{3}^{3}
$$

for $c>0$, where $Q_{1} \neq Q_{2}$.

Letting

$$
E_{1}=\frac{1}{2} E+\frac{\lambda_{4} P r}{3}\|\theta\|_{3}^{3}+\frac{\lambda_{5} P r \epsilon_{T} G_{m}}{3}\left\|\theta^{m}\right\|_{3}^{3}
$$

Article submitted to Royal Society 
and combining (4.2) and (4.3) we now have

$$
\begin{aligned}
\frac{\mathrm{d} E_{1}}{\mathrm{~d} t} & \leq R\left(1+\lambda M_{1}\right)<\theta, w>_{f}-2 \int_{\Omega_{f}} f_{1}|\mathbf{d}|^{2} d \Omega_{f}-\lambda\|\nabla \theta\|_{f}^{2}-\frac{\hat{d}^{3}}{\delta} \int_{\Omega_{m}} f_{2}\left|\mathbf{u}^{m}\right|^{2} d \Omega_{m} \\
& -\frac{2 \hat{d}^{3}}{\phi} \int_{\Omega_{m}} f_{2}\left|\mathbf{d}^{m}\right|^{2} d \Omega_{m}-\lambda \hat{d}^{4}\left\|\nabla \theta^{m}\right\|_{m}^{2}+R^{m} \hat{d}^{3}\left(1+\lambda M_{2} \hat{d}\right)<\theta^{m}, w^{m}>_{m} \\
& +\frac{1}{2} \int_{\Lambda}\left(|\mathbf{u}|^{2} w-\frac{\hat{d}^{3}}{\phi^{2}}\left|\mathbf{u}^{m}\right|^{2} w^{m}\right) d S+\frac{R M_{1} \beta_{1}^{2} \lambda_{4}}{3} \int_{\Omega_{f}}\left|\mathbf{u}^{3}\right| d \Omega_{f} \\
& +\left(\frac{R^{m} M_{2} \beta_{2}^{2} \epsilon_{T}^{\frac{1}{2}} \hat{d}^{\frac{11}{2}} \lambda_{4}}{3}+\frac{2 \Gamma P r \epsilon_{T} \hat{d}^{3}}{3 \delta R^{m} \alpha_{3}^{2}}\right) \int_{\Omega_{m}}\left|\mathbf{u}^{m}\right|^{3} d \Omega_{m} \\
& -\left(\frac{8 \pi^{2} \lambda_{4}}{9}-\frac{2 R M_{1} \lambda_{4}}{3 \beta_{1}}-\frac{2 \Gamma P r \alpha_{1}}{3 R}\right) \int_{\Omega_{f}}|\theta|^{3} d \Omega_{f} \\
& -\left(2 \omega-\frac{4 \Gamma P r}{3 \alpha_{1}^{2} R}\right) \int_{\Omega_{f}}|\mathbf{d}|^{3} d \Omega_{f}-\left(\frac{2 \omega \hat{d}^{5}}{\phi}-\frac{4 \Gamma P r \epsilon_{T} \hat{d}^{3}}{3 \alpha_{2}^{2} R^{m} \phi}\right) \int_{\Omega_{m}}\left|\mathbf{d}^{m}\right|^{3} d \Omega_{m} \\
& -\left(\frac{8 \pi^{2} \lambda_{4} \epsilon_{T}^{\frac{1}{2}} \hat{d}^{\frac{11}{2}}}{9}-\frac{2 R^{m} M_{2} \lambda_{4} \epsilon_{T}^{\frac{1}{2}} \hat{d}^{\frac{11}{2}}}{3 \beta_{2}}-\frac{\Gamma P r \epsilon_{T} \hat{d}^{3}}{3 R^{m}}\left(\frac{2 \alpha_{2}}{\phi}+\frac{\alpha_{3}}{\delta}\right)\right) \int_{\Omega_{m}}\left|\theta^{m}\right|^{3} d \Omega_{m}
\end{aligned}
$$

where $\alpha_{i}, \beta_{j}$ are positive constants for $i=1,2,3 ; j=1,2$ introduced by using Young's inequality on the cubic terms. Before we choose the coefficients to bind the cubic $\mathbf{d}, \theta, \mathbf{d}^{m}$ and $\theta^{m}$ integrals, we must address the boundary integrals and cubic $\mathbf{u}$ and $\mathbf{u}^{m}$ terms in (4.4).

To achieve this we utilise the following Poincaré like inequalities:

$$
\int_{\Omega_{f}}|\mathbf{u}|^{3} d \Omega_{f} \leq c_{1} \int_{\Omega_{f}}|\mathbf{d}|^{3} d \Omega_{f}
$$

and

$$
\int_{\Lambda}|\mathbf{u}|^{3} d S+c_{2} \int_{\Omega_{f}}|\mathbf{u}|^{3} d \Omega_{f} \leq c_{3} \int_{\Omega_{f}}|\mathbf{d}|^{3} d \Omega_{f},
$$

where $c_{1}, c_{2}, c_{3}>0$. Similar inequalities follow in the porous case, for constants $c_{1}^{m}, c_{2}^{m}, c_{3}^{m}$. A proof of these inequalities and the definitions of the constants are 
given in Appendix A. Applying (4.5) and (4.6) to (4.4) yields

$$
\begin{aligned}
\frac{\mathrm{d} E_{1} \leq}{\mathrm{d} t} \leq & R\left(1+\lambda M_{1}\right)<\theta, w>_{f}-2 \int_{\Omega_{f}} f_{1}|\mathbf{d}|^{2} d \Omega_{f}-\lambda\|\nabla \theta\|_{f}^{2}-\frac{\hat{d}^{3}}{\delta} \int_{\Omega_{m}} f_{2}\left|\mathbf{u}^{m}\right|^{2} d \Omega_{m} \\
& -\frac{2 \hat{d}^{3}}{\phi} \int_{\Omega_{m}} f_{2}\left|\mathbf{d}^{m}\right|^{2} d \Omega_{m}-\lambda \hat{d}^{4}\left\|\nabla \theta^{m}\right\|_{m}^{2}+R^{m} \hat{d}^{3}\left(1+\lambda M_{2} \hat{d}\right)<\theta^{m}, w^{m}>_{m} \\
& -\left(\frac{8 \pi^{2} \lambda_{4}}{9}-\frac{2 R M_{1} \lambda_{4}}{3 \beta_{1}}-\frac{2 \Gamma P r \alpha_{1}}{3 R}\right) \int_{\Omega_{f}}|\theta|^{3} d \Omega_{f} \\
& -\left(2 \omega-\frac{4 \Gamma P r}{3 \alpha_{1}^{2} R}-\frac{R M_{1} \beta_{1}^{2} \lambda_{4} c_{1}}{3}-\frac{c_{3}-c_{1} c_{2}}{2}\right) \int_{\Omega_{f}}|\mathbf{d}|^{3} d \Omega_{f} \\
& -\left(\frac{2 \omega \hat{d}^{5}}{\phi}-\frac{2 \Gamma P r \epsilon_{T} \hat{d}^{3}}{3 R^{m}}\left(\frac{2}{\phi \alpha_{2}^{2}}+\frac{c_{1}^{m}}{\delta \alpha_{3}^{2}}\right)-\frac{R^{m} M_{2} \beta_{2}^{2} \epsilon_{T}^{\frac{1}{2}} \hat{d}^{\frac{11}{2}} \lambda_{4} c_{1}^{m}}{3}\right. \\
& \left.-\frac{\hat{d}^{3}\left(c_{3}^{m}-c_{1}^{m} c_{2}^{m}\right)}{2 \phi^{2}}\right) \int_{\Omega_{m}}\left|\mathbf{d}^{m}\right|^{3} d \Omega_{m} \\
& \left.-\left(\frac{8 \pi^{2} \lambda_{4} \epsilon_{T}^{\frac{1}{2}} \hat{d}^{\frac{11}{2}}}{9}-\frac{2 R^{m} M_{2} \lambda_{4} \epsilon_{T}^{\frac{1}{2}} \hat{d}^{\frac{11}{2}}}{3 \beta_{2}}-\frac{\Gamma P r \epsilon_{T} \hat{d}^{3}}{3 R^{m}}\left(\frac{2 \alpha_{2}}{\phi}+\frac{\alpha_{3}}{\delta}\right)\right) \int_{\Omega_{m}}\left|\theta^{m}\right|^{3} d \Omega_{m} .7\right)
\end{aligned}
$$

Now put $\lambda_{4}=\lambda_{4}^{\prime}+k \varepsilon$, and let

$$
\begin{aligned}
& \frac{8 \pi^{2} \lambda_{4}^{\prime}}{9}-\frac{2 R M_{1} \lambda_{4}^{\prime}}{3 \beta_{1}}-\frac{2 \Gamma P r \alpha_{1}}{3 R}=0 \\
& \frac{8 \pi^{2} \lambda_{4}^{\prime} \epsilon_{T}^{\frac{1}{2}} \hat{d}^{\frac{11}{2}}}{9}-\frac{2 R^{m} M_{2} \lambda_{4}^{\prime} \epsilon_{T}^{\frac{1}{2}} \hat{d}^{\frac{11}{2}}}{3 \beta_{2}}-\frac{\Gamma P r \epsilon_{T} \hat{d}^{3}}{3 R^{m}}\left(\frac{2 \alpha_{2}}{\phi}+\frac{\alpha_{3}}{\delta}\right)=0,
\end{aligned}
$$

which is satisfied for

$$
\beta_{2}=\beta_{1}\left(\frac{\epsilon_{T}^{3}}{\hat{d}^{5}}\right)^{\frac{1}{2}}, \quad \frac{2 \alpha_{2}}{\phi}+\frac{\alpha_{3}}{\delta}=2 \alpha_{1} \hat{d}, \quad \lambda_{4}^{\prime}=\frac{3 \Gamma \operatorname{Pr} \alpha_{1} \beta_{1}}{4 \pi^{2} R \beta_{1}-3 R^{2} M_{1}} .
$$

We now minimize

$$
\frac{4 \Gamma P r}{3 \alpha_{1}^{2} R}+\frac{\Gamma P r \alpha_{1} M_{1} \beta_{1}^{2} c_{1}}{4 \pi^{2} \beta_{1}-3 R M_{1}}
$$

with respect to $\alpha_{1}$ and $\beta_{1}$ to yield

$$
\alpha_{1}=\frac{8 \pi^{2} 4^{\frac{1}{3}}}{9 R M_{1} c_{1}^{\frac{1}{3}}}, \quad \beta_{1}=\frac{9 R M_{1}}{8 \pi^{2}},
$$

and choose $\alpha_{3}$ to minimize

$$
\frac{2 \Gamma P r \epsilon_{T} \hat{d}^{3}}{3 R^{m}}\left(\frac{2}{\phi \alpha_{2}^{2}}+\frac{c_{1}^{m}}{\delta \alpha_{3}^{2}}\right) .
$$

Article submitted to Royal Society 
From (4.7), choosing $k=27 /\left(8 \pi^{2}\right)$ we can now deduce

$$
\begin{aligned}
\frac{\mathrm{d} E_{1} \leq}{\mathrm{d} t} \leq & \left.R\left(1+\lambda M_{1}\right)<\theta, w\right\rangle_{f}-2 \int_{\Omega_{f}} f_{1}|\mathbf{d}|^{2} d \Omega_{f}-\lambda\|\nabla \theta\|_{f}^{2}-\frac{\hat{d}^{3}}{\delta} \int_{\Omega_{m}} f_{2}\left|\mathbf{u}^{m}\right|^{2} d \Omega_{m} \\
& -\frac{2 \hat{d}^{3}}{\phi} \int_{\Omega_{m}} f_{2}\left|\mathbf{d}^{m}\right|^{2} d \Omega_{m}-\lambda \hat{d}^{4}\left\|\nabla \theta^{m}\right\|_{m}^{2}+R^{m} \hat{d}^{3}\left(1+\lambda M_{2} \hat{d}\right)<\theta^{m}, w^{m}>_{m} \\
& -\varepsilon \int_{\Omega_{f}}|\theta|^{3} d \Omega_{f}-\hat{\omega} \int_{\Omega_{f}}|\mathbf{d}|^{3} d \Omega_{f}-\hat{\omega}^{m} \int_{\Omega_{m}}\left|\mathbf{d}^{m}\right|^{3} d \Omega_{m}-\varepsilon \epsilon_{T}^{\frac{1}{2}} \hat{d}^{\frac{11}{2}} \int_{\Omega_{m}}\left|\theta^{m}\right|^{3} d \Omega_{m},
\end{aligned}
$$

where we require

$$
\begin{aligned}
\hat{\omega}= & 2 \omega-\frac{81 \Gamma \operatorname{Pr} 4^{\frac{1}{3}} R M_{1}^{2} c_{1}^{\frac{2}{3}}}{64 \pi^{4}}-\frac{c_{3}-c_{1} c_{2}}{2}-\varepsilon \frac{729 R^{3} M_{1}^{3} c_{1}}{512 \pi^{6}}>0 \\
\hat{\omega}^{m}= & \frac{2 \omega \hat{d}^{5}}{\phi}-\frac{2 \Gamma \operatorname{Pr} \epsilon_{T} \hat{d}^{3}}{3 R^{m}}\left(\frac{2}{\phi \alpha_{2}^{2}}+\frac{c_{1}^{m}}{\delta \alpha_{3}^{2}}\right)-\frac{27 \Gamma \operatorname{Pr} 4^{\frac{1}{3}} R^{m} M_{2}^{2} c_{1}^{m} \epsilon_{T}^{\frac{5}{2}} \hat{d}^{\frac{3}{2}}}{32 \pi^{4} c_{1}^{\frac{1}{3}}} \\
& -\frac{\hat{d}^{3}\left(c_{3}^{m}-c_{1}^{m} c_{2}^{m}\right)}{2 \phi^{2}}-\varepsilon \frac{729\left(R^{m}\right)^{3} M_{2}^{3} c_{1}^{m} \epsilon_{T}^{\frac{1}{2}} \hat{d}^{\frac{11}{2}}}{512 \pi^{6}}>0 .
\end{aligned}
$$

Defining

$$
\begin{aligned}
\mathcal{I}= & R\left(1+\lambda M_{1}\right)<\theta, w>_{f}+R^{m} \hat{d}^{3}\left(1+\lambda M_{2} \hat{d}\right)<\theta^{m}, w^{m}>_{m}, \\
\mathcal{D}= & 2 \int_{\Omega_{f}} f_{1}|\mathbf{d}|^{2} d \Omega_{f}+\lambda\|\nabla \theta\|_{f}^{2}+\frac{\hat{d}^{3}}{\delta} \int_{\Omega_{m}} f_{2}\left|\mathbf{u}^{m}\right|^{2} d \Omega_{m} \\
& +\frac{2 \hat{d}^{3}}{\phi} \int_{\Omega_{m}} f_{2}\left|\mathbf{d}^{m}\right|^{2} d \Omega_{m}+\lambda \hat{d}^{4}\left\|\nabla \theta^{m}\right\|_{m}^{2},
\end{aligned}
$$

it follows that

$$
\frac{\mathrm{d} E_{1}}{\mathrm{~d} t} \leq-\mathcal{D}\left(\frac{R_{E}-1}{R_{E}}\right)-\varepsilon \int_{\Omega_{f}}|\theta|^{3} d \Omega_{f}-\varepsilon \epsilon_{T}^{\frac{1}{2}} \hat{d}^{\frac{11}{2}} \int_{\Omega_{m}}\left|\theta^{m}\right|^{3} d \Omega_{m}
$$

where

$$
\frac{1}{R_{E}}=\max _{\mathcal{H}}\left(\frac{\mathcal{I}}{\mathcal{D}}\right)<1
$$

Utilising Poincaré like inequalities (cf. Payne \& Straughan 1998) allows us to deduce that

$$
\frac{\mathrm{d} E_{1}}{\mathrm{~d} t} \leq-m E_{1}
$$

where $m>0$. Integrating, we have $E_{1}(t) \leq E_{1}(0) \mathrm{e}^{-m t} \rightarrow 0$ as $t \rightarrow \infty$, where convergence is at least exponential, so we have established unconditional nonlinear stability provided (4.8) and (4.9) hold.

The corresponding Euler Lagrange equations which arise at the sharpest threshold $R_{E}=1$ are

$$
\begin{gathered}
2 f_{1} \nabla^{2} u_{i}+2 f_{1}^{\prime} w_{, i}+2 f_{1}^{\prime} u_{i, 3}+k_{i} R\left(M_{1} \lambda+1\right) \theta=L, i \\
2 \lambda \nabla^{2} \theta+R\left(M_{1} \lambda+1\right) w=0
\end{gathered}
$$


in the fluid layer, and

$$
\begin{gathered}
2 \delta \hat{d}^{3}\left(f_{2} \nabla^{2} u_{i}^{m}+f_{2}^{\prime} w_{, i}^{m}+\phi f_{2}^{\prime} u_{i, 3}^{m}\right)-2 \phi \hat{d}^{3} f_{2} u_{i}^{m}+k_{i} \phi \delta R^{m} \hat{d}^{3}\left(M_{2} \lambda \hat{d}+1\right) \theta^{m}=\phi \delta L_{, i}^{m} \\
2 \lambda \hat{d} \nabla^{2} \theta^{m}+R^{m}\left(M_{2} \lambda \hat{d}+1\right) w^{m}=0
\end{gathered}
$$

in the porous layer, where $L$ and $L^{m}$ are Lagrange multipliers.

By taking the double curl of equations $(4.10)_{1}$ and $(4.11)_{1}$ and adopting normal mode representations, the twelfth-order eigenvalue problem (4.10) - (4.11) can be utilised to locate the critical nonlinear Rayleigh number $R a_{E}$, which is given by

$$
R a_{E}=\max _{\lambda} \min _{a^{2}} R^{2}\left(a^{2}, \lambda\right) .
$$

Numerical results for the nonlinear energy approach are presented in $\S 5$.

\section{Results and conclusions}

The first key result that can be derived is for the case $\Gamma=0$, which corresponds to the viscosity of the fluid being constant with respect to temperature. Under this condition the equations for linear instability and nonlinear stability are identical to those of Hill \& Straughan (2009), for which excellent agreement was shown between the two. It is important to note, though, that in this paper the nonlinear advection term $\mathbf{v} \cdot \nabla \mathbf{v}$ is included in the analysis, whereas the analysis of Hill \& Straughan (2009) is limited to the nonlinear Stokes problem.

For $\Gamma \neq 0$ we now solve the eigenvalue problem (4.10) - (4.11) by means of a $D^{2}$ Chebyshev tau method. The details are similar to those given by Dongarra et al. (1996). The parameters, unless stated otherwise, are fixed at $\delta=5 \times 10^{-6}$, $G_{m}=10, \operatorname{Pr}=6$ and $\epsilon_{T}=0.7$. The porous material is assumed to be that of a Foametal (which is used extensively in industrial applications such as heat exchangers, chemical reactors and fluid filters), with physical values of permeability and porosity of $8.19 \times 10^{-8} \mathrm{~m}^{2}$ and 0.79 , respectively (cf. Straughan 2002, Goyeau et al. 2003).

Figure 1 shows the neutral curves for a variation of $\Gamma$ values, where the linear instability and nonlinear stability thresholds are represented by solid and dashed lines respectively.

It is clear that an increase in $\Gamma$ causes the system to become more stable. Assuming that the temperature at the boundaries remains fixed, this corresponds to the strength of the linear dependence of viscosity on temperature increasing. As the viscosity decreases with an increase in temperature, this physcially makes sense. An interesting result is that the bimodal nature of the neutral curve is unaffected by the change in $\Gamma$.

Since the linear instability and nonlinear stability results clearly show excellent agreement, we can conclude that the linear theory accurately encapsulates the physics of the onset of convection.

\section{Appendix A. A proof of inequalities (4.5) and (4.6)}

Let $\Omega_{f}$ represent the fluid period cell, and $u_{i} \in C^{1}$ be a solenoiodal function satisfying the boundary condition $u_{i}=0$ on $\Lambda_{0}$, where the boundary of $\Omega_{f}$ is given by 


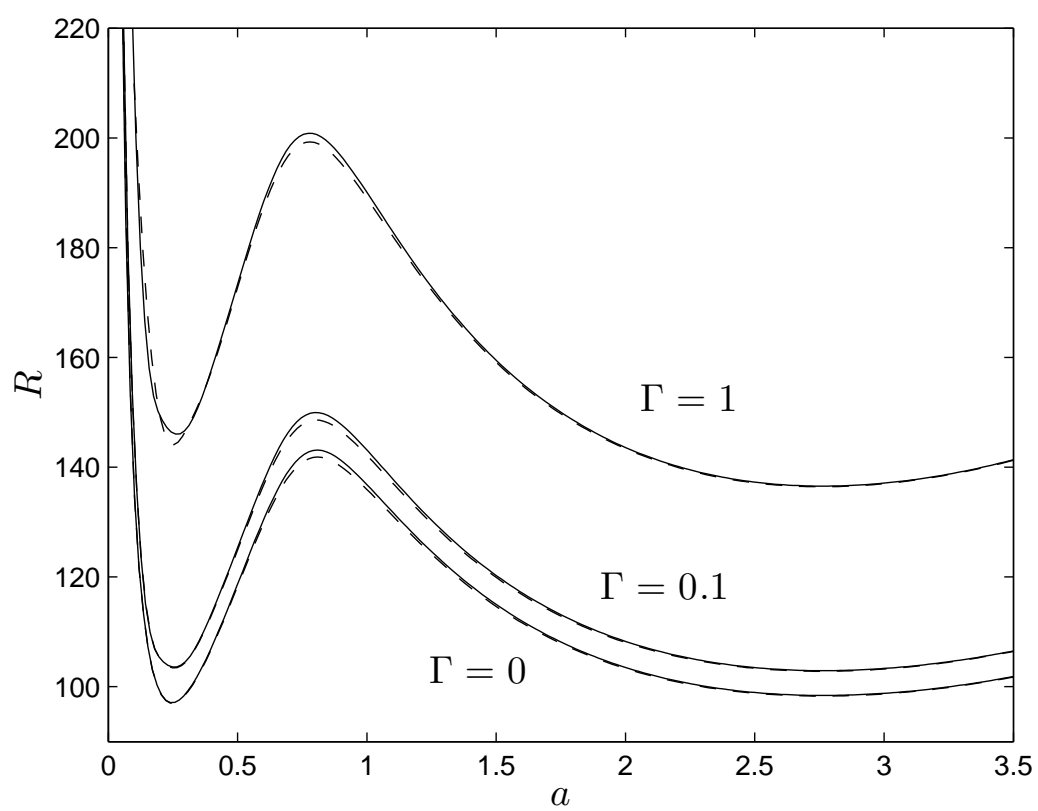

Figure 1. Visual representation of linear instability (solid lines) and nonlinear stability (dashed lines) thresholds, with critical thermal Rayleigh number $R$ plotted against wavenumber $a$, for $\Gamma=0,0.1$ and 1 . The remaining parameters are $\hat{d}=0.116, \operatorname{Pr}=6$, and $\phi=0.79$.

$\partial \Omega_{f}=\Lambda+\Lambda_{0}$. In the same vein as $\S 4, \Lambda$ represents the fluid/porous interface at $z$ $=0$. For a $C^{1}$ function $f_{i}$ to be chosen at our discretion, we observe that

$$
\int_{\Lambda} f_{i} n_{i}|\mathbf{u}|^{3} d S=\int_{\Omega_{f}} f_{i, i}|\mathbf{u}|^{3} d \Omega_{f}+3 \int_{\Omega_{f}} f_{i}|\mathbf{u}| u_{j} u_{j, i} d \Omega_{f}
$$

and

$$
\begin{aligned}
& 3 \int_{\Lambda} f_{i} n_{j}|\mathbf{u}| u_{j} u_{i} d S=3 \int_{\Omega_{f}} f_{i}|\mathbf{u}|, j u_{j} u_{i} d \Omega_{f} \\
& \quad+3 \int_{\Omega_{f}} f_{i}|\mathbf{u}| u_{j} u_{i, j} d \Omega_{f}+3 \int_{\Omega_{f}} f_{i, j}|\mathbf{u}| u_{j} u_{i} d \Omega_{f}
\end{aligned}
$$

By letting

$$
\mathbf{f}=\left(-p_{1} x,-p_{1} y,-p_{1} z-p_{2}\right),
$$

where $p_{1}$ and $p_{2}$ are constants to be chosen at our discretion, it follows that

$$
\begin{aligned}
& p_{2} \int_{\Lambda}|\mathbf{u}|^{3} d S-3 \int_{\Lambda} f_{i} u_{i}|\mathbf{u}| w d S+6 p_{1} \int_{\Omega_{f}}|\mathbf{u}|^{3} d \Omega_{f} \\
& \quad=3 \int_{\Omega_{f}} f_{i}|\mathbf{u}| u_{j}\left(u_{i, j}+u_{j, i}\right) d \Omega_{f}+3 \int_{\Omega_{f}} f_{i}|\mathbf{u}|_{, j} u_{j} u_{i} d \Omega_{f} .
\end{aligned}
$$

Article submitted to Royal Society 
The arithmetic-geometric mean inequality leads us to

$$
\int_{\Lambda} x_{\beta} u_{\beta} w|\mathbf{u}| d S \leq \frac{d_{1}}{2}\left(\frac{1}{\alpha} \int_{\Lambda} w^{2}|\mathbf{u}| d S+\alpha \int_{\Lambda} u_{\beta}^{2}|\mathbf{u}| d S\right)
$$

where $\alpha>0$ is a constant, and $d_{1}=\max _{i=1,2}\left(1,\left|x_{i}\right|\right)$. Employing this inequality yields

$$
\begin{aligned}
& \left(4 p_{2}-\frac{3 p_{1} d_{1}}{2 \alpha}\right) \int_{\Lambda} w^{2}|\mathbf{u}| d S+\left(p_{2}-\frac{3 p_{1} d_{1} \alpha}{2}\right) \int_{\Lambda} u_{\beta}^{2}|\mathbf{u}| d S+6 p_{1} \int_{\Omega_{f}}|\mathbf{u}|^{3} d \Omega_{f} \\
& \leq 3 \int_{\Omega_{f}} f_{i}|\mathbf{u}| u_{j}\left(u_{i, j}+u_{j, i}\right) d \Omega_{f}+3 \int_{\Omega_{f}} f_{i}|\mathbf{u}|, j u_{j} u_{i} d \Omega_{f} .
\end{aligned}
$$

Letting $\alpha=1 / 2$ and $p_{2}=3 p_{1} d_{1} / 4$ to remove the boundary integrals, we now have

$$
6 p_{1} \int_{\Omega_{f}}|\mathbf{u}|^{3} d \Omega_{f} \leq \frac{63 p_{1} d_{1}}{4} \int_{\Omega_{f}}|\mathbf{u}|^{2}|\mathbf{d}| d \Omega_{f}
$$

By using the Cauchy-Schwartz inequality on the right hand side, we find

$$
\int_{\Omega_{f}}|\mathbf{u}|^{3} d \Omega_{f} \leq\left(\frac{21 d_{1}}{8}\right)^{3} \int_{\Omega_{f}}|\mathbf{d}|^{3} d \Omega_{f},
$$

which is inequality (4.5).

To derive inequality (4.6) we return to (A 1). By letting

$$
p_{2}=\frac{p_{1} d_{1}}{2 \alpha}\left(1-\alpha^{2}\right),
$$

and using (4.5) it follows that

$$
\int_{\Lambda}|\mathbf{u}|^{3} d \Lambda+\frac{12 \alpha}{d_{1}\left(1-4 \alpha^{2}\right)} \int_{\Omega_{f}}|\mathbf{u}|^{3} d \Omega_{f} \leq \frac{9\left(21 d_{1}\right)^{2}\left(2 \alpha-\alpha^{2}+1\right)}{64\left(1-4 \alpha^{2}\right)} \int_{\Omega_{f}}|\mathbf{d}|^{3} d \Omega_{f}
$$

as required, where $\alpha$ is a constant to be chosen at our discretion.

\section{References}

Alazmi, B. \& Vafai, K. 2000 Analysis of variants within the porous media transport models. ASME J. Heat Transfer 122, 303-326.

Alazmi, B. \& Vafai, K. 2001 Analysis of fluid flow and heat transfer interfacial conditions between a porous medium and a fluid layer. Int. J. Heat Mass transfer 44, 1735-1749.

Antontsev, S., Diaz, J. \& Shmarev, S. 2001 Energy methods for free boundary problems. Boston: Birkhauser.

Blest, C., Duffy, B. R., Mckee, S. \& Zulkfle, A. K. 1999 Curing simulation of thermoset composites. Composites, Part A 30, 1289-1309.

Capone, F. \& Gentile, M. 1994 Nonlinear stability analysis of convection for fluids with exponentially temperature-dependent viscosity. Acta Mech. 107, 53-64.

Capone, F. \& Gentile, M. 1995 Nonlinear stability analysis of the Benard problem for fluids with a convex nonincreasing temperature depending viscosity. Continuum Mech. Thermodyn. 7, 297-309. 
Carr, M. 2004 Penetrative convection in a superposed porous-medium-fluid layer via internal heating. J. Fluid Mech. 509, 305-329.

Chang, M. H. 2004 Stability of convection induced by selective absorption of radiation in a fluid overlying a porous layer. Phys. Fluids 16, 3690-3698.

Chang, M. H. 2005 Thermal convection in superposed fluid and porous layers subjected to a horizontal plane Couette flow. Phys. Fluids 17, 064106-1-064106-7.

Chang, M. H. 2006 Thermal convection in superposed fluid and porous layers subjected to a plane Poiseuille flow. Phys. Fluids 18, 035104-1-035104-10.

Chen, F. \& Chen, C. F. 1988 Onset of finger convection in a horizontal porous layer underlying a fluid layer. J. Heat Transfer 110, 403-409.

Dongarra, J. J., Straughan, B. \& Walker, D.W. 1996 Chebyshev tau-QZ algorithm methods for calculating spectra of hydrodynamic stability problems. App. Num. Math. 22, 399-434.

Ewing, E. \& Weekes, S. 1998 Numerical methods for contaminant transport in porous media. Comput. Math. 202, 75-95.

Galiano, G. 2000 Spatial and time localization of solutions of the Boussinesq system with nonlinear thermal diffusion. Nonlin. Analysis 42, 423-438.

Goyeau, B., Lhuillier, D., Gobin, D. \& Velarde M. G. 2003 Momentum transport at a fluid-porous interface. Int. J. Heat Mass Trans. 46, 4071-4081.

Hill, A. A. \& Straughan, B. 2009 Global stability for thermal convection in a fluid overlying a highly porous material. Proc Roy Soc A 465, 207-217.

Hirata, S. C., Goyeau, B., Gobin, D., Carr, M. \& Cotta, R. M. 2007 Linear stability of natural convection in superposed fluid and porous layers: influence of the interfacial modelling. Int. J. Heat Mass Transfer 50, 1356-1367.

Hoppe, R. H. W., Porta, P. \& Vassilevski, Y. 2007 Computational issues related to iterative coupling of subsurface and channel flows. Calcolo 44, 1-20.

Ladyzhenskaya, O. A. 1967 New equations for the description of motions of viscous incompressible fluids and global solvability of their boundary value problems. Tr. Mat. Inst. Steklova 102, 85-104.

Ladyzhenskaya, O. A. 1968 On some nonlinear problems in the theory of continuous media. Am. Math. Soc. Transl. 2 70, 73-89.

Ladyzhenskaya, O. A. 1969 The mathematical theory of viscous incompressible flow, 2nd edn. New York: Gordon and Breach.

Manga, M., Weeraratne, D. \& Morris, S. J. S. 2001 Boundary-layer thickness and instabilities in Bénard convection of a liquid with a temperature-dependent viscosity. Phys. Fluids 13, 802-805.

Mu, M. \& Xu, J.C. 2007 A two-grid method of a mixed Stokes - Darcy model for coupling fluid flow with porous medium flow. SIAM J. Numer. Anal. 45, 1801-1813.

Nield, D. A. \& Bejan, A. 2006 Convection in porous media, 3rd edn. New York: SpringerVerlag.

Payne, L. E. \& Straughan, B. 1998 Analysis of the boundary condition at the interface between a viscous fluid and a porous medium and related modelling questions. J. Math. Pures Appl. 77, 317-354.

Payne, L. E. \& Straughan, B. 2000 Unconditional nonlinear stability in temperaturedependent viscosity flow in a porous medium. Stud. Appl. Math. 105, 59-81.

Payne, L. E., Song, J. C. \& Straughan, B. 1999 Continuous dependence and convergence results for Brinkman and Forchheimer models with variable viscosity. Proc. Roy. Soc. London A 455, 2173-2190.

Shevtsova, V. M., Melnikov, D. E. \& Legros, J. C. 2001 Three-dimensional simulations of hydrodynamic instability in liquid bridges: Influence of temperature-dependent viscosity. Phys. Fluids 13, 2851-2865.

Article submitted to Royal Society 
Straughan, B. 2002 Sharp global nonlinear stability for temperature-dependent viscosity convection. Proc Roy Soc A 458, 1773-1782.

Straughan, B. 2004 The energy method, stability and nonlinear convection. New York: Springer.

Straughan, B. 2008 Stability and wave motion in porous media. Appl. Math. Sci. Ser. vol. 165. New York: Springer.

Vafai, K. 2005 Handbook of Porous Media. 2nd edition, New York: Taylor \& Francis.

Vafai, K. \& Thiyagaraja, R. 1987 Analysis of flow and heat transfer at the interface region of a porous medium. Int. J. Heat Mass transfer 30, 1391-1405.

Article submitted to Royal Society 